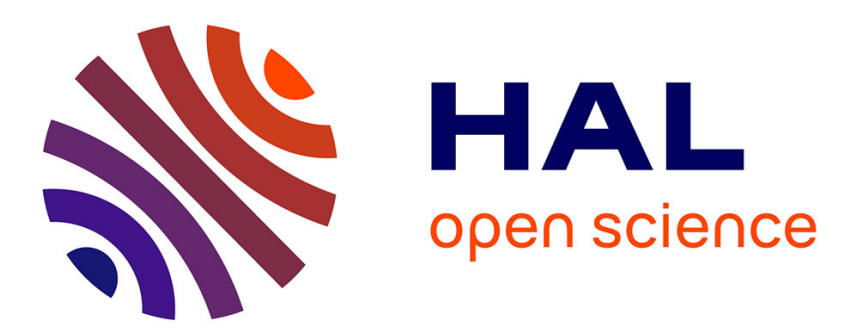

\title{
Comparaison des relations entre l'éducation parentale et les performances scolaires dans deux milieux socio-culturels contrastés (populaire et non populaire)
}

Youssef Tazouti, André Flieller, Pierre Vrignaud

\section{- To cite this version:}

Youssef Tazouti, André Flieller, Pierre Vrignaud. Comparaison des relations entre l'éducation parentale et les performances scolaires dans deux milieux socio-culturels contrastés (populaire et non populaire). Revue Française de Pédagogie, 2005. hal-02343803

\section{HAL Id: hal-02343803 \\ https://hal.univ-lorraine.fr/hal-02343803}

Submitted on 3 Nov 2019

HAL is a multi-disciplinary open access archive for the deposit and dissemination of scientific research documents, whether they are published or not. The documents may come from teaching and research institutions in France or abroad, or from public or private research centers.
L'archive ouverte pluridisciplinaire HAL, est destinée au dépôt et à la diffusion de documents scientifiques de niveau recherche, publiés ou non, émanant des établissements d'enseignement et de recherche français ou étrangers, des laboratoires publics ou privés. 


\section{Comparaison des relations entre l'éducation parentale et les performances scolaires dans deux milieux socio-culturels contrastés (populaire et non populaire) *}

Youssef Tazouti, André Flieller, Pierre Vrignaud

La plupart des recherches sur les relations entre l'éducation familiale et les performances scolaires reposent sur le postulat, implicite ou explicite, que ces relations sont indépendantes du milieu socio-culturel. La présente recherche s'intéresse au contraire à l'existence d'éventuelles différences dans ces relations entre les milieux populaire et non populaire. L'hypothèse a été mise à l'épreuve au moyen d'une analyse secondaire de l'enquête 20 ans après (Flieller, Manciaux \& Kop, 1995). Un modèle explicatif des performances scolaires a été construit en fonction des variables disponibles dans cette enquête. La conformité des données au modèle théorique a été testée par des analyses en pistes causales effectuées à l'aide des logiciels AMOS et LISREL, d'une part sur l'échantillon global (492 élèves de CE1 et leurs familles) et, d'autre part, sur deux souséchantillons contrastés (246 familles populaires et 246 familles non populaires). Les résultats montrent l'adéquation des données au modèle, qui explique $59 \%$ de la variance des performances scolaires. Mais surtout, la comparaison de l'échantillon populaire et de l'échantillon non populaire à l'aide d'un modèle d'équations structurales, révèle que le réseau de relations entre les variables du modèle présente des différences significatives entre les deux milieux. II se pourrait que l'influence du milieu socio-culturel sur les performances scolaires passe davantage par l'école pour les enfants du milieu populaire, et davantage par l'éducation familiale pour les enfants de milieu non populaire. Cette interprétation des résultats demande à être confirmée par de nouvelles recherches.

Descripteurs (TEE) : éducation par la famille, milieu culturel, milieu social, réussite scolaire.

Méthodologie : analyses en pistes causales, modèles d'équations structurales.

* Nous remercions Michel Manciaux, professeur émérite de santé publique à l'université Henri Poincaré-Nancy 1 et Jean-Luc Kop, maître de conférences de méthodologie de la psychologie à l'université Nancy 2 , qui, avec l'un d'entre nous, ont effectué l'enquête 20 ans après d'où sont tirées les données de la présente recherche. 


\section{INTRODUCTION}

De nombreuses recherches ont établi que les pratiques éducatives varient selon le milieu socio-culturel des parents (cf. par ex. la revue de Palacio-Quintin, 1995 ou la recherche récente de Bradley et al., 2001 sur un large échantillon représentatif de la population des États-Unis). L'étude des relations entre l'éducation familiale et les performances scolaires des enfants s'en trouve compliquée, car elle doit, d'une manière ou d'une autre, tenir compte du milieu socioculturel des parents. Celui-ci peut en effet agir sur les performances scolaires de l'enfant par d'autres biais que l'éducation familiale stricto sensu. En dépit des critiques dont elles ont fait l'objet, on peut se reporter à ce propos à l'explication sociologique proposée par Bourdieu et Passeron (1970), qui repose sur la formation et les effets d'un habitus pénalisant les élèves d'origine sociale défavorisée, ou à l'explication sociolinguistique avancée par Bernstein (1975), qui oppose le code restreint des familles socialement défavorisées au code élaboré requis par l'école et familier aux enfants socialement favorisés. II en résulte que I'on ne peut attribuer à l'éducation parentale un rôle dans les performances scolaires des enfants qu'à la condition de contrôler le milieu socio-culturel.

Trois stratégies sont observées dans la littérature. La première consiste à traiter l'éducation familiale comme une variable intermédiaire entre le milieu socio-culturel et les performances scolaires. Dans les études qui l'adoptent, on suppose que les performances scolaires dépendent de l'éducation familiale et non pas directement du milieu socio-culturel, mais on admet que l'éducation diffère selon les milieux. En d'autres termes, l'éducation est considérée comme une variable proximale, et le milieu social, comme une variable distale. Cette approche est celle de Lautrey (1980). En se fondant principalement sur les règles de vie en vigueur dans la famille, l'auteur distingue trois types de structuration de l'environnement familial (souple, rigide et aléatoire), qui sont inégalement favorables au développement cognitif et aux performances scolaires de l'enfant. Mais ces types de structuration sont aussi inégalement distribués selon les milieux, la plus favorable (structuration souple) s'observant plus fréquemment dans les milieux favorisés. Des conclusions similaires se dégagent de recherches nord-américaines utilisant l'échelle HOME (1) pour mesurer l'environnement familial : le développement cognitif et/ou les performances scolaires dépendent de l'éducation familiale et celle-ci, du milieu socio-culturel (Palacio-Quintin, 1995). Notons cepen- dant que beaucoup d'études se contentent de corrélations entre paires de variables, ce qui est insuffisant pour prouver que l'éducation familiale constitue une variable intermédiaire. Les recherches seraient plus convaincantes si elles montraient que la corrélation entre le milieu socio-culturel et les performances scolaires s'annule ou s'atténue fortement quand on contrôle l'éducation au moyen de méthodes statistiques appropriées (corrélations partielles, régression multiple pas à pas, pistes causales...). La recherche de Muller (1990), l'une des premières à utiliser le modèle LISREL (2) dans ce domaine, est à cet égard très innovante. Celle de Barocas et al. (1991), constitue un deuxième exemple de recherche méthodologiquement bien conduite : à l'aide d'un modèle d'équations structurales, elle montre que la variable " style d'enseignement de la mère " exerce un effet sur le développement cognitif de l'enfant, qui n'est pas réductible à celui du milieu socio-économique.

La deuxième stratégie, moins fréquente que la précédente, consiste à étudier les relations entre éducation familiale et performances scolaires au sein d'un même milieu social, en général le milieu populaire. En principe, les relations établies ainsi ne peuvent pas être attribuées au milieu socio-culturel puisqu'il est maintenu constant. Deux variantes principales peuvent être distinguées. Dans la première, on oppose les familles où l'enfant réussit normalement ses études à celles où il rencontre des difficultés scolaires. Ayant dichotomisé l'échantillon sur cette base, on compare les pratiques éducatives parentales dans les deux sous-échantillons et l'on repère ainsi celles qui sont favorables à la réussite scolaire. C'est ainsi que Espéret (1979) compare l'éducation parentale d'élèves de sixième de même milieu, à la fois social et culturel (enfants d'ouvriers ayant le certificat d'études primaires au maximum), mais placés, les uns dans une filière d'excellence $(n=61)$, les autres dans une filière de rattrapage ou de relégation $(n=41)$. Zéroulou (1988) compare de la même manière quinze familles algériennes, où l'un des enfants au moins a fait des études supérieures, à quinze autres familles de même origine, où aucun enfant n'a accédé au cycle secondaire long. Dans leur recherche effectuée en Belgique dans des milieux pauvres (parents chômeurs ou percevant l'équivalent belge du RMI), Pourtois et al. (1992) comparent les caractéristiques personnelles (par ex. aptitudes intellectuelles, motivation scolaire), familiales (par ex. style éducatif, attitude envers l'école) et environnementales (par ex. représentations des enseignants) de trente enfants en situation d'échec en fin d'école primaire et de vingt-neuf enfants de même milieu mais ayant accompli une 
scolarité primaire normale. La recherche de Rochex (1995), qui compare les projets parentaux et les conversations familiales sur l'école selon la réussite et l'adaptation scolaire de quelques adolescents de milieu social défavorisé observés intensivement, participe de cette même démarche. La seconde variante de la deuxième stratégie présente deux différences par rapport à la précédente. D'une part, elle reconnaît que le milieu socio-culturel que l'on étudie n'est pas parfaitement homogène et qu'il existe d'inévitables différences de statut socio-culturel entre les familles, qu'il est nécessaire de contrôler. D'autre part, plutôt que de remonter de la variable dépendante (les performances scolaires) vers la variable indépendante (l'éducation familiale), elle préfère se donner les moyens d'étudier directement l'influence de celle-ci sur celle-là. Cette approche est initiée par Tazouti (2003) qui, dans une recherche récente, teste, au moyen d'un modèle d'équations structurales, l'influence de l'éducation familiale sur les performances scolaires en milieu populaire (élèves de CE2, $N=128$ ). Les résultats de ce travail confirment partiellement I'hypothèse selon laquelle l'influence du milieu socioculturel sur les performances scolaires est médiatisée par l'éducation familiale.

La troisième stratégie combine les deux premières tout en s'en distinguant sur un point fondamental. Comme dans la première stratégie, on considère que l'influence du milieu socio-culturel sur les performances scolaires est médiatisée par l'éducation familiale. Comme dans la seconde, on étudie un réseau de causalité à partir d'observations faites à milieu social constant. En revanche, et contrairement aux deux précédentes approches, on adme t ici, à titre d'hypothèse, que ce réseau de causalité puisse partiellement différer d'un milieu à l'autre. En d'autres termes, on considère que l'influence de telle ou telle pratique éducative parentale sur les performances scolaires de l'enfant peut différer selon le milieu socio-culturel considéré. On trouve dans la littérature quelques indications directes ou indirectes en faveur de cette hypothèse. Sur des enfants québécois de quatre ans, Palacio-Quintin et Jourdan-Ionescu (cités par PalacioQuintin 1995) constatent que la corrélation entre l'environnement familial mesuré par le HOME et le développement cognitif est plus importante dans le milieu socio-culturel le plus défavorisé. Steinberg, Lamborn, Dornbusch \& Darling (1992) mettent en relation le style éducatif familial et les performances scolaires de 6400 adolescents américains. Ils observent que le style "démocratique " est associé à de meilleures performances chez les enfants blancs, mais pas chez les enfants afro-américains ou asiatiques dont la réussite scolaire est associée au contraire au style "autoritaire ". Une étude récente de Marjoribanks (2003) révèle que les écarts de performances scolaires entre les enfants de niveau intellectuel et d'environnement familial différents varient selon l'origine sociale : l'écart est plus important dans le milieu socio-culturel moyen que dans le milieu socio-culturel élevé. Deux études françaises fournissent des indications plus indirectes, mais intéressantes néanmoins, car elles suggèrent que la réussite scolaire peut être associée à des pratiques éducatives différentes. D'un côté, Prêteur \& Louvet-Schmauss (1994) constatent des différences de pratiques éducatives au sein du milieu social favorisé qui, en grande section de maternelle, ne se traduisent pas par des différences de performances scolaires, également bonnes chez les enfants de parents "fonctionnalistes " et chez ceux de parents " attentistes ". D'un autre côté, l'étude de Laurens (1992) sur 167 enfants d'ouvriers ayant intégré une école d'ingénieurs montre que l'excellence scolaire dépend de l'éducation parentale, mais qu'il n'existe pas une forme unique d'éducation menant à l'excellence ; l'auteur en distingue au contraire trois, correspondant chacune à environ un tiers des familles observées. Si l'on ajoute que Marjoribanks (2001) ne trouve pas, à onze ans, les mêmes relations entre l'environnement familial et les performances scolaires chez les filles $(n=266)$ et chez les garçons $(n=250)$ (3), on est amené à penser que les relations entre l'éducation familiale et les performances scolaires sont peut-être plus complexes qu'on ne l'a envisagé. II se pourrait en particulier que la réussite scolaire soit associée à des contextes éducatifs familiaux différents selon le milieu socio-culturel. Les parents des enfants qui ont de bonnes performances scolaires ont-ils les mêmes pratiques éducatives ? Au delà d'un certain nombre de régularités, la valorisation de l'école par exemple, il est fort possible que l'on observe certaines différences d'un milieu socio-culturel à l'autre, par exemple dans la forme de l'accompagnement scolaire ou du soutien à la motivation scolaire.

C'est cette hypothèse générale que nous avons voulu mettre à l'épreuve dans la présente recherche. Le test de l'hypothèse nécessite de disposer d'un effectif de parents et d'enfants suffisamment important et de pouvoir opérer un contrôle statistique de l'environnement scolaire. Aussi notre travail reposet-il sur une analyse secondaire des données de l'enquête 20 ans après (Flieller, Manciaux, \& Kop, 1994 \& 1995), qui satisfait à ces deux conditions. L'objectif principal de cette enquête était d'étudier l'évolution des compétences cognitives des écoliers, leurs capa- 
cités de raisonnement en particulier, sur une période de vingt ans. Deux cohortes d'écoliers, observées au même âge de sept ans et avec les mêmes instruments en 1973 et en 1992 ont été comparées. Les données de 1973 proviennent d'une enquête de I'INSERM (Manciaux, 1975) effectuée sur la totalité des enfants nés et scolarisés dans le département de Meurthe-et-Moselle. Les instruments d'observation utilisés en 1992 ont été repris sans changement de l'enquête précédente et appliqués à un échantillon de 2334 enfants de sept ans du même département, ainsi qu'à leur famille. Un sous-échantillon aléatoire de 531 familles a fait l'objet d'une observation plus approfondie au moyen d'un questionnaire comportant des items nouveaux ou repris d'enquêtes antérieures. Ce sont les données recueillies sur ce souséchantillon, limité aux élèves de CE1 et à leurs parents (4), qui ont été analysées dans la perspective indiquée ci-dessus.

\section{MODĖLE THÉORIQUE}

Pour comparer les relations entre l'éducation familiale et les performances scolaires dans deux milieux socio-culturels contrastés, il convient de partir d'un modèle théorique général. Nous avons construit ce modèle explicatif des performances scolaires sur la base d'une part des connaissances théoriques disponibles, et, d'autre part des variables figurant dans l'enquête 20 ans après.

Ce modèle est présenté dans la figure 1 . On voit qu'il fait intervenir douze variables relatives 1) au milieu socio-culturel; 2) à l'éducation parentale ; 3) aux aptitudes cognitives de l'enfant et 4) à son environnement pédagogique. Nous verrons plus loin, dans la partie méthodologique, ce que recouvrent précisément ces variables et comment elles ont été opérationnalisé es. Remarquons pour l'instant que le milieu socio-culturel et l'environnement pédagogique constituent des variables explicatives considérées comme corrélées, la distribution des élèves de milieux socio-culturels différents dans les diverses écoles ne pouvant pas être tenue pour aléatoire. Le milieu socio-culturel exerce des effets directs et indirects sur les performances scolaires. Bien entendu, ce sont les effets indirects, médiatisés principalement par l'éducation familiale, qui nous intéressent le plus dans cette recherche. Cependant, la sociologie de l'éducation (cf. par ex., Duru-Bellat \& Henriot-Van Zanten, 1992) ne permettant pas d'attribuer tous les effets du milieu socio-culturel à la seule éducation parentale, le modèle prévoit un effet direct. L'éducation parentale est décrite par huit variables qui sont en relation plus ou moins proches avec l'école et les performances scolaires. L'accompagnement parental de la scolarité par exemple constitue une variable proximale, tandis que les valeurs éducatives générales (valorisation de l'autonomie vs valorisation du conformisme) constituent des variables distales, censées agir indirectement sur les performances scolaires, via les attentes des parents envers l'école d'une part et leur style éducatif d'autre part. Remarquons enfin que le modèle fait jouer un rôle aux aptitudes intellectuelles de l'enfant et les considère comme déterminées en partie par trois facteurs: le milieu socio-culturel (effet direct), l'éducation parentale et - dans une perspective vygostkienne des rapports entre le développement cognitif et les apprentissages scolaires - l'environnement pédagogique. Les performances scolaires constituent évidemment la variable dépendante ultime.

\section{MÉTHODOLOGIE}

\section{Participants et déroulement de l'enquête}

Les participants sont constitués des 492 élèves de CE1 et de leurs familles observées intensivement dans l'enquête 20 ans après à l'aide du questionnaire approfondi. II faut toutefois préciser que les élèves considérés ici se trouvaient en CE1 lors de la mesure des variables indépendantes du modèle théorique et en début de CE2 lors de la mesure des performances scolaires (cf. infra "Procédure de recueil des données ").

\section{Mesure du niveau économico-culturel et construction des sous-échantillons}

Le niveau économico-culturel des familles a été mesuré par un indice agrégeant quatre indicateurs : 1) le niveau d'instruction de la mère; 2) le niveau d'instruction du père ; 3 ) les revenus pondérés du ménage, calculés selon la formule : revenu mensuel du ménage / (nombre d'adultes + 0,5 nombre d'enfants) ; 4) l'espace disponible dans le logement, à savoir le nombre de pièces du logement divisé par le nombre de personnes habitant le ménage.

Les corrélations entre les indicateurs sont comprises entre 0,36 et 0,59 . Une analyse en composantes principales permet de ne retenir qu'un seul facteur expliquant $63 \%$ de la variance totale. Les saturations des indicateurs dans ce 


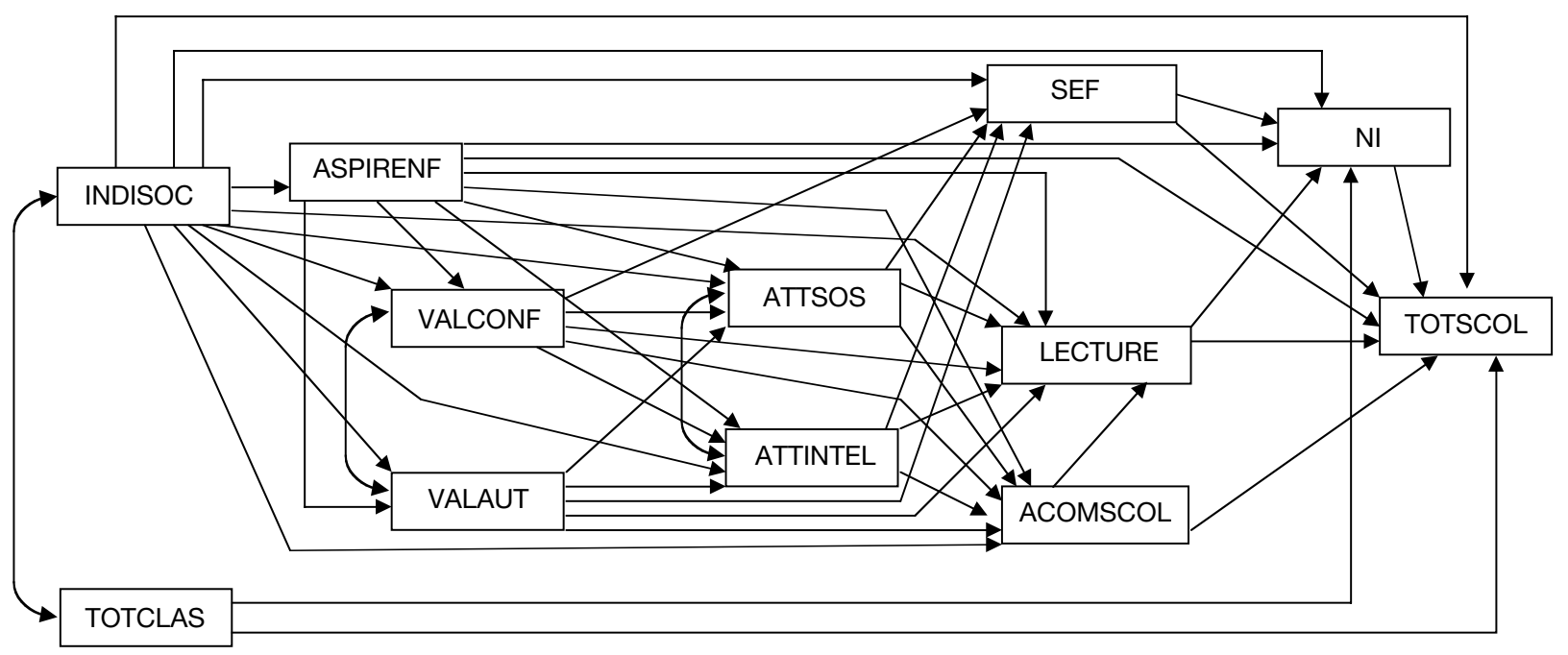

INDISOC : indice économico-culturel

VALAUT : valeurs d'autonomie en éducation

ATTSOS : attentes de socialisation envers l'école

SEF : style éducatif familial

LECTURE : rapport de l'enfant à la lecture

NI : niveau intellectue
ASPIRENF : aspirations parentales pour la scolarité de l'enfant

VALCONF : valeurs de conformisme en éducation

ATTINTEL : attentes intellectuelles envers l'école

ACOMSCOL : accompagnement parental de la scolarité de l'enfant

TOTCLAS : environnement pédagogique

TOTSCOL : performances scolaires

Note de lecture : un rectangle représente une variable; une flèche unidirectionnelle représente l'effet causal d'une variable indépendante sur une variable dépendante (effet mesuré par un coefficient de piste) ; une double flèche représente une relation entre deux variables pour laquelle on ne fait pas d'hypothèse de causalité.

\section{Figure 1. - Modèles en pistes causales théorique}

facteur sont élevées: 0,87 pour le revenu pondéré du ménage, 0,80 pour le niveau d'instruction de la mère, 0,77 pour celui du père et 0,72 pour l'espace disponible. Ces résultats montrent qu'il est légitime d'agréger les indicateurs. La mesure ainsi obtenue est continue et définit un gradient d'appartenance sociale.

La moyenne de l'indice économico-culturel $(\mathrm{m}=2,26)$ a permis de dichotomiser l'échantillon en deux souséchantillons de même effectif : d'une part, les 246 familles présentant un indice inférieur à la moyenne, que l'on a considérées comme appartenant au milieu «populaire », et d'autre part, les 246 autres familles, considérées comme appartenant au milieu « non populaire ». La moyenne de l'indice est évidemment très différente dans les deux sous-échantillons $\left(\mathrm{m}_{1}=\right.$ 1,36 et $\left.m_{2}=3,17\right)$, mais elle ne permet pas à elle seule de juger du contraste obtenu. La comparaison des appartenances socio-professionnelles et des niveaux d'instruction dans les deux milieux apporte des précisions utiles. On constate ainsi que :

- 68,5 \% des pères de l'échantillon populaire sont des ouvriers, alors que $59,5 \%$ de ceux de l'échantillon non populaire appartiennent aux PCS les plus élevées (cadres supérieurs, professions libérales et professions intermédiaires);

- $59 \%$ des mères de l'échantillon populaire sont au foyer et $15 \%$ occupent des emplois d'ouvrières ou de personnels de service alors que l'échantillon non populaire comporte $31 \%$ de mères au foyer et $30 \%$ de PCS supérieures ;

- $88,5 \%$ des pères et $89 \%$ des mères de l'échantillon populaire ont un niveau d'étude inférieur ou égal au CAP, tandis que $59,5 \%$ des pères et $56 \%$ des mères de l'échantillon non populaire ont un niveau d'étude égal ou supérieur au baccalauréat. 
Les deux sous-échantillons sont donc fortement contrastés. On note que leur homogénéité interne, reflétée par la variance de l'indice économico-culturel, est également différente, le sous-échantillon populaire s'avérant plus homogène que l'autre. (5)

\section{Mesure de l'environnement pédagogique}

La mesure de l'environnement pédagogique correspond à la différence entre les performances attendues et les performances réelles des élèves de la classe. Les performances attendues sont celles que l'appartenance socio-culturelle des élèves permet de prévoir, tandis que les performances réelles sont celles obtenues aux épreuves nationales d'évaluation de rentrée en CE2.

Le calcul qui permet de l'obtenir comporte cinq étapes : 1) on régresse la moyenne des performances scolaires sur la moyenne des indices économico-culturels, la classe étant l'unité statistique de cette régression ; 2) on calcule la performance moyenne des élèves de la classe fréquentée par l'enfant (Mo), celui-ci n'intervenant pas dans le calcul ; 3) on calcule de même la moyenne des indices économico-culturels des élèves de la classe ; 4) à l'aide de l'équation de régression obtenue à l'étape 1 , on calcule la moyenne attendue des performances scolaires de la classe (Ma), compte tenu de l'indice économico-culturel moyen de la classe ; 5) on calcule la différence (Mo - Ma). Une différence positive indique un environnement favorable, puisque les élèves de la classe obtiennent aux épreuves nationales des performances supérieures à celles statistiquement attendues en fonction de leur milieu socio-culturel. Une différence négative indique au contraire un environnement défavorable. La mesure obtenue est continue.

Tel qu'il est opérationnalisé, l'environnement pédagogique prend en compte l'ensemble des facteurs internes à la classe et susceptibles d'influer sur les performances des élèves qui s'y trouvent placés : effectif de la classe, niveau de la classe, efficacité pédagogique de l'enseignant, climat social, etc. II ne se réduit donc pas à «l'effet maître ».

\section{Mesure de l'éducation familiale}

Le modèle comporte huit variables éducatives: 1) la valeur accordée par les parents à l'autonomie de l'enfant ; 2) celle qu'ils accordent au respect des normes sociales et familiales par l'enfant; 3) les attentes des parents envers l'école au plan de la socialisation ; 4) leurs attentes envers l'école au plan cognitif ; 5) le style éducatif familial : variable mesurée par des items portant sur des pratiques éducatives encourageant l'autonomie de l'enfant et dont des études antérieures (Aubret-Bény, 1978 ; Manciaux, 1975) ont montré qu'elles étaient corrélées au développement cognitif (pour des informations détaillées, voir Flieller, Manciaux \& Kop, 1995, p. 256-258); 6) l'accompagnement parental de la scolarité de l'enfant ; 7) le rapport de l'enfant à la lecture (que l'on suppose fortement influencé à cet âge par l'éducation familiale) ; 8) le niveau d'aspiration scolaire des parents pour l'enfant (niveau d'études souhaité).

À l'exception de la dernière qui est mesurée par un item unique, chacune de ces huit variables est mesurée par une échelle constituée de trois à sept items (au total, trente-deux items du questionnaire approfondi). Ces sept échelles composites sont répertoriées dans le tableau $n^{\circ} 1$ qui indique, pour chacune, le nombre d'items, l'homogénéité interne de l'épreuve (évaluée par le coefficient alpha de Cronbach) et le libellé d'un item pris comme exemple.

Le tableau fait apparaître que l'homogénéité interne des échelles est satisfaisante, compte tenu du nombre réduit d'items les composant, à l'exception des échelles de " style éducatif familial » et d' «accompagnement parental de la scolarité de l'enfant » qui présentent des coefficients alpha relativement faibles (respectivement 0,42 et 0,48 ).

\section{Mesure du niveau intellectuel de l'enfant}

Le niveau intellectuel de l'enfant a été mesuré au moyen du cahier II de l'Échelle collective de niveau intellectuel (ECNI) mise au point par Bénédetto (1969). L'épreuve comporte huit épreuves, dont quatre verbales et quatre non verbales. Ces épreuves font appel principalement au raisonnement inductif. Toutefois, les études de validation ont montré que le score total à l'ECNI était fortement corrélé à des épreuves classiques telles que le WISC (6).

\section{Mesure des performances scolaires}

Les performances scolaires en français et en mathématiques ont été mesurées en début de CE2 dans le cadre de l'évaluation de rentrée. Rappelons que cette évaluation, coordonnée par la Direction de l'évaluation et de la prospective du ministère de l'Éducation nationale, a lieu tous les ans et que les épreuves sont élaborées à l'échelon national. Nous n'avons pas distingué ici les scores de français et de mathématiques. La variable "performances scolaires » est donc un score composite donnant un poids égal aux deux disciplines. 
Tableau I. - Analyse d'items et exemples d'items des différents instruments de mesure de l'éducation familiale

\begin{tabular}{|c|c|c|c|}
\hline Échelles & $\begin{array}{l}\text { Nombre } \\
\text { d'items }\end{array}$ & $\begin{array}{c}\text { Coefficient } \\
\text { alpha }\end{array}$ & Exemple d'items \\
\hline 1) Valeurs d'autonomie en éducation & 4 & .57 & $\begin{array}{l}\text { Importance de l'initiative, de l'esprit critique } \\
\text { (échelle en quatre points) }\end{array}$ \\
\hline 2) Valeurs de conformisme en éducation & 4 & .73 & $\begin{array}{l}\text { Importance de l'obéissance, de la politesse } \\
\text { (idem) }\end{array}$ \\
\hline 3) Attentes de socialisation envers l'école & 6 & .68 & $\begin{array}{l}\text { L'école doit apprendre aux élèves à vivre } \\
\text { en groupe (accord?) }\end{array}$ \\
\hline 4) Attentes intellectuelles envers l'école & 4 & .55 & $\begin{array}{l}\text { L'école doit apprendre aux élèves la lecture } \\
\text { et l'écriture (idem) }\end{array}$ \\
\hline 5) Style éducatif familial & 7 & .42 & $\begin{array}{l}\text { Votre enfant peut-il intervenir dans la } \\
\text { conversation des adultes? }\end{array}$ \\
\hline $\begin{array}{l}\text { 6) Accompagnement parental de la scolarité } \\
\text { de l'enfant }\end{array}$ & 3 & .48 & $\begin{array}{l}\text { Vérifiez-vous le travail scolaire fait par votre } \\
\text { enfant à la maison? }\end{array}$ \\
\hline 7) Rapport de l'enfant à la lecture & 3 & .60 & $\begin{array}{l}\text { Votre enfant lit-il à la maison des revues } \\
\text { pour enfant? }\end{array}$ \\
\hline
\end{tabular}

Note de lecture : Le coefficient alpha de Cronbach est un indice de fidélité qui se rapporte aux fluctuations dues au contenu de l'échelle. $\mathrm{Sa}$ valeur dépend à la fois des intercorrélations entre les items, et donc de leur homogénéité, mais aussi de leur nombre. On considère en général qu'une valeur supérieure à .70 témoigne d'une bonne fidélité du score à l'échelle (cf. la revue de Peterson, 1994). Néanmoins, des valeurs inférieures peuvent être considérées comme acceptables lorsque l'indice, comme c'est le cas ici, est utilisé comme une mesure d'homogénéité des items et que l'échelle est courte.

\section{Procédure de recueil des données}

Le test de niveau intellectuel a été administré collectivement par des psychologues scolaires au mois de février. Parallèlement, des renseignements généraux sur l'enfant et sa famille ont été recueillis au moyen d'un questionnaire distribué aux parents par les écoles. Le questionnaire approfondi a été envoyé en mai et en juin aux parents sélectionnés par le tirage au sort. Ce questionnaire a été rempli de manière anonyme par les parents et renvoyé directement à l'équipe de recherche (une clé numérique a permis d'assembler ultérieurement les fichiers). Enfin, les résultats à l'évaluation nationale d'entrée en CE2 ont été communiqués en septembre à l'équipe de recherche avec l'autorisation de l'administration de l'Éducation nationale.

\section{RÉSULTATS}

Nous présenterons les résultats en deux parties : l'une, assez brève, concernera la mise à l'épreuve du modèle théorique sur l'ensemble de l'échantillon ; l'autre, plus développée, portera sur la comparaison du réseau de relations entre variables dans le milieu populaire et dans le milieu non populaire. Les résultats doivent permettre de répondre à trois questions : 1) le modèle théorique est-il satisfaisant? 2) le réseau de causalité entre les variables de ce modèle diffère-t-il significativement d'un milieu à l'autre? 3) si oui, où se situent les différences principales ? Cette partie présente uniquement les réponses aux trois questions précédentes ; on trouvera les commentaires et interprétations des résultats dans la partie suivante. En exposant les résultats, nous serons obligés de donner quelques indications sur les procédures d'analyse utilisées (7). Des informations plus détaillées sont présentées dans l'annexe méthodologique de l'article.

La mise à l'épreuve du modèle théorique et la comparaison des relations entre variables dans les deux milieux repose sur des modèles statistiques dits Modèles d'équations structurales (MES). Ces modèles permettent à la fois d'évaluer l'adéquation des données au modèle théorique et d'estimer l'importance des relations entre les variables. L'adéquation globale des données au modèle théorique est 
évaluée à partir d'un ensemble d'indices présentés dans l'annexe. La significativité statistique des relations permet d'en juger de manière plus fine. Les relations entre les variables du modèle s'expriment par des coefficients appelés " coefficients de piste " qui mesurent l'effet d'une variable indépendante sur une variable dépendante. Ces coefficients sont estimés par la méthode du maximum de vraisemblance. Après standardisation, ils varient de -1 à +1 (voir l'annexe pour plus de détails). Deux types d'effet d'une variable $X$ sur une variable $Y$ sont à considérer : d'une part l'effet direct de $X$ sur $Y$, et d'autre part l'effet indirect ou médiatisé, qui résulte de l'effet de $X$ sur des variables $Z_{1}$, $Z_{2} \ldots Z_{n}$ exerçant elles-mêmes un effet sur $Y$ (cf. l'annexe pour des informations sur le mode de calcul des effets indirects).

\section{Test du modèle théorique sur l'ensemble de l'échantillon}

Les paramètres du modèle théorique et les indices d'adéquation ont été estimés au moyen du logiciel AMOS version 5 (Arbuckle \& Wothke, 1999 ; Arbuckle, 2003). Les données manquantes, par exemple les résultats aux épreuves de l'évaluation nationale des élèves ayant changé d'école à l'issue du CE1, ont été estimées par un algorithme d'imputation (Wothke \& Arbuckle, 1996). L'adéquation des données au modèle est correcte puisque tous les indices d'adéquation ont une valeur proche de celle considérée habituellement comme satisfaisante $\left(\chi^{2}=40,06\right.$ pour 20 degrés de liberté, $\mathrm{p}=.005 ; \mathrm{RMSEA}=0,045 ; \mathrm{TLI}=0,94$; se reporter à l'annexe pour une définition de ces indices et la manière de les interpréter). Au total, le modèle rend compte de $59 \%$ de la variance des performances scolaires.

A titre de contrôle, le modèle théorique a été testé par un autre logiciel permettant également l'estimation des paramètres des MES, à savoir le logiciel LISREL, version 8.50 (Jöreskog \& Sörbom, 1998). Les deux logiciels fournissent des coefficients de piste très proches (8). Les indices d'adéquation sont également satisfaisants $\left(\chi^{22}=35\right.$ pour 20 degrés de liberté, $p=0,02$; RMSEA $=0,04 ; \mathrm{GFI}=0,99$; $A G F I=$ 0,95 ; cf. Annexe pour la signification de ces indices). Les variables du modèle théorique permettent d'expliquer ici encore $59 \%$ de la variance des performances scolaires.

La réponse à la première question est donc positive : l'adéquation des données au modèle théorique est satisfaisante. Toutefois, les relations prévues par le modèle ne sont pas toutes confirmées. Par exemple, l'influence directe du style éducatif familial sur les performances scolaires de l'enfant ne s'avère pas significative. II en va de même pour l'influence directe de l'accompagnement scolaire parental sur les performances de l'enfant. Au total, quinze pistes causales sur les quarante-trois comportées par le modèle (Figure 1) ne sont pas significatives au seuil de 0,05 . Nous n'irons pas plus loin dans cette ana- lyse, dans la mesure où c'est la comparaison des relations causales dans les deux milieux sociaux qui nous intéresse ici et où la pertinence du modèle théorique est dans l'ensemble assurée.

\section{Comparaison des relations entre variables dans les deux sous-échantillons}

\section{Constats}

Les figures 2 et 3 présentent les coefficients de pistes estimés par AMOS dans chacun des deux sous-échantillons pris séparément (9). Pour une meilleure lisibilité, seules les pistes significatives à .05 sont tracées dans ces schémas. De son côté, le tableau 2 compare les effets directs, indirects et totaux sur les performances scolaires des principales variables indépendantes.

Plusieurs constatations peuvent être faites. D'une part, l'adéquation des données au modèle est bonne dans chaque échantillon, ce que confirme l'estimation par LISREL (10). D'autre part, les figures et le tableau font apparaître un certain nombre de différences entre les deux milieux.

(1) Le pourcentage de la variance des performances scolaires expliquée par le modèle est un peu plus élevé pour le milieu populaire (51\%) que pour le milieu non populaire (47\%).

(2) Certains coefficients de piste sont significatifs dans un échantillon mais pas dans l'autre, ce qui se traduit par des flèches présentes dans une figure et pas dans l'autre. C'est ainsi par exemple que les revenus et le niveau d'instruction des parents (mesurés par l'indice économico-culturel) exercent un effet positif et significatif sur l'accompagnement parental de la scolarité de l'enfant dans les familles populaires (Figure 2, coefficient de piste $=0,27$ ) qui ne se retrouve pas dans les familles de l'autre milieu (Figure 3). Mais à l'inverse, le style éducatif des parents a un effet significatif sur le niveau intellectuel de l'enfant dans le milieu non populaire (Figure 3 : coefficient de piste $=0,21$ ), mais pas dans le milieu populaire (Figure 2). II apparaît ainsi que les variables indépendantes du modèle n'ont pas toutes les mêmes effets sur les variables dépendantes dans les deux milieux sociaux comparés.

(3) On relève également des différences intermilieux pour la plupart des variables exerçant un effet appréciable sur les performances scolaires, à l'exception de l'accompagnement scolaire parental (cf. Tableau 2). L'effet de l'indice économico-culturel est 


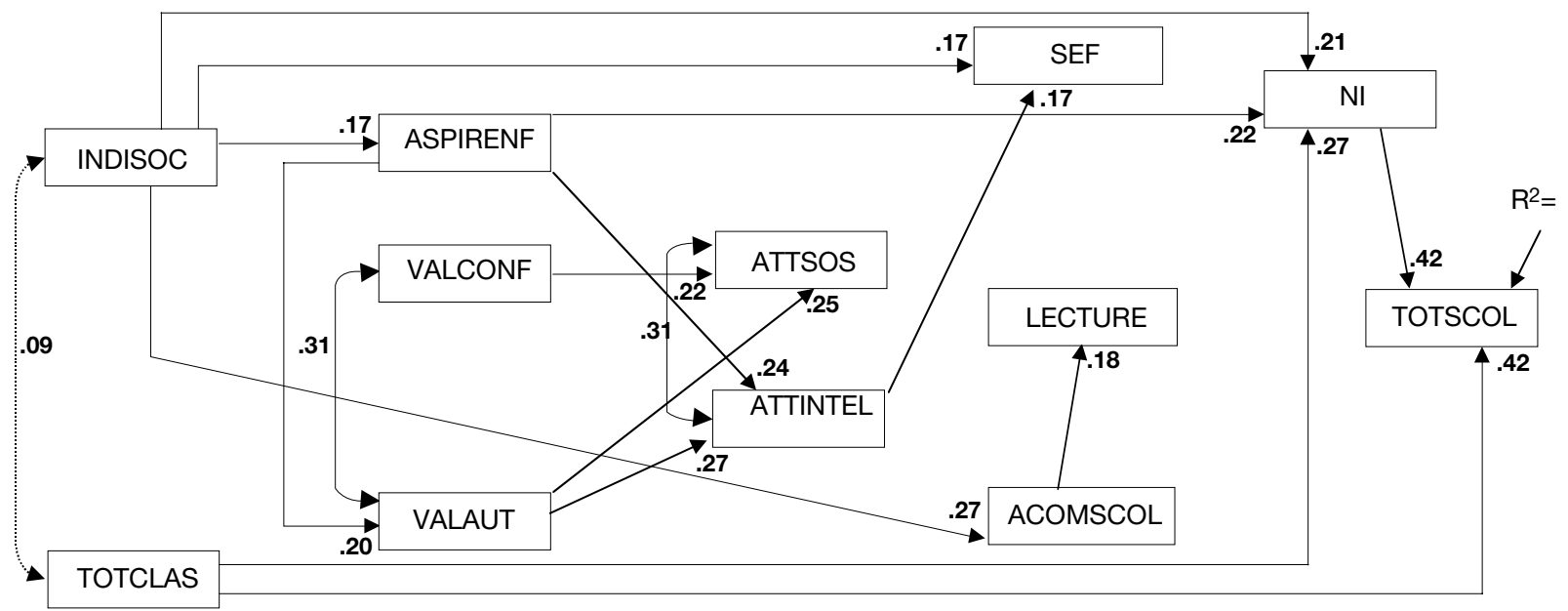

Note de lecture : Les valeurs indiquées sur les flèches unidirectionnelles sont les valeurs standardisées des coefficients de piste. Elles indiquent de quelle proportion d'écart-type la variable dépendante augmente lorsque la variable indépendante augmente d'un écarttype. Par exemple, la valeur .42 sur la flèche qui joint le niveau intellectuel (NI) aux performances scolaires (TOTSCOL) signifie que les performances scolaires croissent de .42 écart-type quand le niveau intellectuel croît d'un écart-type. Les valeurs sur les doubles flèches sont les valeurs des corrélations estimées par le logiciel.

Figure 2. - Coefficients de piste standardisés estimés par AMOS sur l'échantillon populaire $(\mathbf{N}=\mathbf{2 4 6})$ Modèle non contraint Seules les pistes significatives à .05 sont indiquées

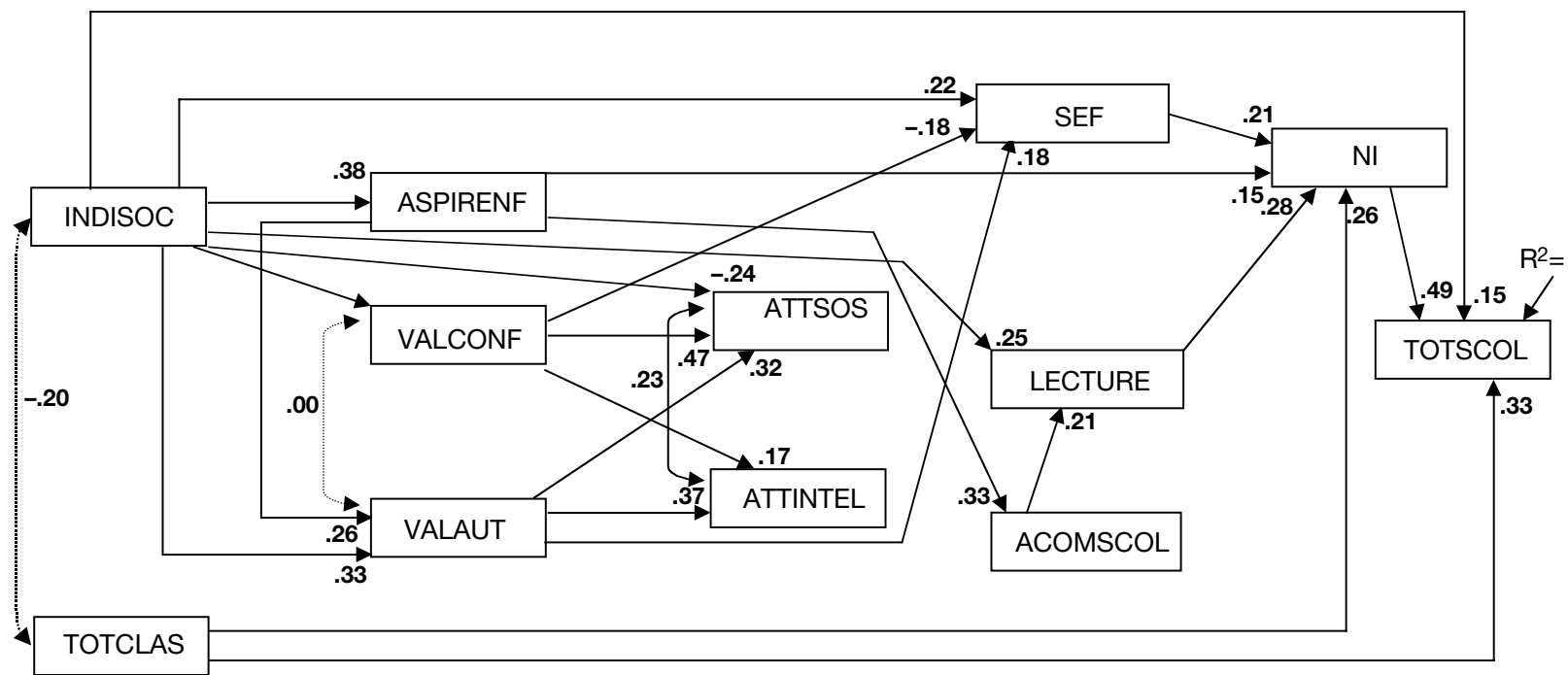

Note de lecture : Les valeurs indiquées sur les flèches unidirectionnelles sont les valeurs standardisées des coefficients de piste. Elles indiquent de quelle proportion d'écart-type la variable dépendante augmente lorsque la variable indépendante augmente d'un écarttype. Par exemple, la valeur .49 sur la flèche qui joint le niveau intellectuel (NI) aux performances scolaires (TOTSCOL) signifie que les performances scolaires croissent de .49 écart-type quand le niveau intellectuel croît d'un écart-type. Les valeurs sur les doubles flèches sont les valeurs des corrélations estimées par le logiciel.

Figure 3. - Coefficients de piste standardisés estimés par AMOS sur l'échantillon populaire $(\mathbf{N}=\mathbf{2 4 6})$ Modèle non contraint Seules les pistes significatives à .05 sont indiquées 
Tableau II. - Effets standardisés directs et indirects des variables du modèle sur les performances scolaires

\begin{tabular}{|l|c|c|c|c|c|c|}
\hline \multirow{2}{*}{\multicolumn{1}{|c|}{ Variables }} & \multicolumn{2}{c|}{ Populaire (N = 246) } & \multicolumn{2}{c|}{ Non populaire (N = 246) } \\
\cline { 2 - 7 } & \multicolumn{3}{|c|}{ Effets } & \multicolumn{3}{c|}{ Effets } \\
\cline { 2 - 7 } & Directs & Indirects & Totaux & Directs & Indirects & Totaux \\
\hline Indice économico-culturel & .07 & .12 & .19 & .15 & .16 & .31 \\
Environnement pédagogique & .42 & .11 & .53 & .33 & .13 & .46 \\
Aspirations parentales pour la scolarité de l'enfant & -.04 & .11 & .07 & .08 & .14 & .22 \\
Accompagnement parental de la scolarité de l'enfant & .07 & .02 & .09 & .07 & .03 & .10 \\
Rapport de l'enfant à la lecture & .08 & .04 & .12 & -.01 & .14 \\
Niveau intellectuel & .42 & 0 & .42 & .49 & 0.13 \\
\hline
\end{tabular}

Note de lecture : Les effets directs correspondent aux coefficients de piste qui indiquent de quelle proportion d'écart-type augmente la variable dépendante lorsque la variable dépendante augmente d'un écart-type. Par exemple (ligne 2, colonne 1), les performances scolaires augmentent de 0,42 écart-type en milieu populaire quand la valeur de l'environnement pédagogique augmente d'un écart-type. C'est l'effet direct, dont la valeur peut être lue sur la figure 2. Mais à cet effet, il faut ajouter celui qui est médiatisé par le niveau intellectuel dit effet « indirect » (voir Figure 2). Pour le calculer, on parcourt le chemin en allant de l'environnement pédagogique au niveau intellectuel » (coefficient de piste $=.27$ ) puis du niveau intellectuel aux performances scolaires (coefficient de piste $=.42$ ) et l'on effectue le produit des deux coefficients : $.27 \times .42=.11$. L'effet total est évidemment la somme des effets directs et indirects, soit dans l'exemple $: .42+.11=.53$.

plus élevé pour le sous-échantillon non populaire $(0,31)$ que pour l'échantillon populaire $(0,19)$, différence due principalement aux effets directs $(0,15$ et 0,07 respectivement). À l'inverse, l'environnement pédagogique exerce un effet un peu plus important sur les performances scolaires des enfants de milieu populaire $(0,54)$ que sur ceux de milieu non populaire $(0,46)$, différence qui, ici aussi, est imputable principalement aux effets directs $(0,43$ et 0,33 respectivement). Les effets directs du rapport à la lecture semblent plus importants dans le milieu populaire $(0,08)$ que dans le milieu non populaire $(-0,01)$, tandis que l'inverse est vrai pour les effets indirects, qui sont plus importants dans le milieu non populaire $(0,14)$ que dans le milieu populaire $(0,04)$. Enfin, l'effet direct du niveau intellectuel de l'enfant sur ses performances scolaires apparaît un peu plus élevé dans le milieu non populaire $(0,49)$ que dans le milieu populaire $(0,42)$.

Au total, ces résultats confirment l'hypothèse centrale de notre recherche, à savoir l'existence de différences inter-milieux dans le réseau de causalité considéré. La réponse à la deuxième question est donc positive. Les figures 2 et 3 et le tableau 2 commentés ci-dessus fournissent des éléments de réponse à la question 3 sur la localisation de ces différences. II reste cependant à tester la significativité statistique de ces différences, ce que nous allons faire à présent.

\section{Comparaisons statistiques}

La comparaison des équations structurales dans deux échantillons à l'aide d'un MES repose sur deux estimations successives des coefficients de piste. Dans la première, les coefficients sont estimés de manière totalement indépendantes dans les deux échantillons (modèle non contraint). Dans la seconde, ils sont estimés sous la condition de recevoir la même valeur dans les deux échantillons (modèle contraint). On considère que les estimations diffèrent d'un échantillon à l'autre si elles sont significativement moins bonnes dans le modèle contraint que dans le modèle non contraint (se reporter à l'Annexe pour une explication plus complète).

La comparaison par AMOS des modèles contraint et non contraint révèle une différence significative : modèle non contraint : $\chi^{2}$ à 40 degrés de liberté $=51,41 ; p=0,11$; modèle contraint : $\chi^{2}$ à 83 degrés de liberté $=173,82$; $p<0,001$. On observe donc une chute de l'adéquation, dont on teste la significativité par différence entre les $\chi^{2}$ de chacun des modèles : on obtient un $\chi^{2}$ à 43 degrés de liberté valant 122,42 dont la probabilité est très faible $(p<0,001)$. On peut donc rejeter l'hypothèse d'égalité de l'ensemble des pistes dans les deux sous-échantillons. 
À titre de vérification, nous avons partagé l'échantillon en deux sous-échantillons aléatoires que nous avons comparés de la même manière. La différence des $\chi^{2}$ des deux modèles - contraint et non contraint - vaut 40,27 (43 ddl, $p=0,59)$. On ne peut donc pas rejeter l'hypothèse d'égalité des pistes entre ces deux sous-échantillons constitués de manière aléatoire. Cette analyse confirme que les différences entre les valeurs de piste dans l'échantillon "populaire " et dans l'échantillon " non populaire » ne sont pas dues au hasard. On peut cependant s'interroger sur la pertinence de l'indicateur économico-culturel dans ces comparaisons intermilieux. En effet, l'indicateur sert à dichotomiser l'échantillon, mais il demeure présent dans les modèles testés. Nous avons donc refait la comparaison des équations structurales entre les deux milieux, mais en supprimant l'indicateur économico-culturel dans les divers modèles. Cette nouvelle comparaison mène à la même conclusion : les coefficients de piste diffèrent d'un milieu à l'autre (différence entre les $\chi^{2}$ des modèles non contraint et contraint : 94,$98 ; 40$ ddl, $p<.001$ ). La robustesse du résultat paraît donc assurée.

Il apparaît donc clairement que le réseau de causalité diffère significativement d'un milieu à l'autre. Cette comparaison peut-être affinée. Après cette comparaison globale, il est en effet possible de comparer la valeur des coefficients dans les deux milieux, piste par piste (cf. l'annexe pour des informations sur le test statistique employé). Le tableau 3 donne la liste des onze pistes pour lesquelles les coefficients sont significativement différents d'un milieu à l'autre (11), ce qui permet de répondre plus avant à la troisième question (localisation des différences).

Cinq de ces pistes concernent l'indice économicoculturel dont l'effet sur l'accompagnement parental de la scolarité et sur le niveau intellectuel de l'enfant est plus important dans les familles populaires $(0,27)$ que dans les familles non populaires $(-0,02 \mathrm{~ns})$, tandis que l'inverse s'observe pour la valorisation de l'autonomie de l'enfant et le rapport de l'enfant à la lecture, variables pour lesquelles l'effet est plus important dans les familles non populaires $(0,33$ et 0,25 respectivement) que dans les familles populaires $(-0,03 \mathrm{~ns}$ et $-0,05$ ns respectivement); en ce qui concerne les attentes de socialisation par l'école, l'effet de l'indice change de sens d'un milieu à l'autre : il est positif pour les familles populaires $(0,12)$ mais négatif pour les familles non populaires $(-0,24)$. Les aspirations scolaires des parents (niveau d'étude espéré pour l'enfant) distinguent également les deux milieux, avec un effet plus important sur la valorisation de l'autonomie en milieu non populaire $(0,26$ contre 0,20$)$ et, surtout, sur l'accompagnement scolaire $(0,33$ contre $0,03 \mathrm{~ns})$. Des effets plus importants en milieu non populaire s'observent aussi pour la valorisation de la conformité sur les attentes de socialisation par l'école $(0,47$ contre 0,22$)$, le style éducatif parental sur le niveau intellectuel de l'enfant $(0,21$ contre $-0,12 \mathrm{~ns})$,

\section{Tableau III. - Comparaison des coefficients de piste standardisés (beta) par AMOS (les valeurs sont celles du modèle non contraint)}

(cf. figure 1 pour la signification des étiquettes des variables)

\begin{tabular}{|c|c|c|c|c|}
\hline \multirow{2}{*}{ VI } & \multirow{2}{*}{ VD } & \multicolumn{2}{|c|}{ Valeurs des pistes } & \multirow{2}{*}{$\begin{array}{l}\text { Significativité } \\
\text { de la différence } \\
\left({ }^{\star *}=.01 ;{ }^{*}=.05\right)\end{array}$} \\
\hline & & Populaire & Non populaire & \\
\hline INDSOC & VALAUT & -.03 & .33 & ** \\
\hline INDSOC & ATTSOS & .12 & -.24 & ** \\
\hline INDSOC & ACOMSCOL & .27 & -.02 & ** \\
\hline INDSOC & LECTURE & -.05 & .25 & ** \\
\hline INDSOC & $\mathrm{NI}$ & .21 & .12 & ** \\
\hline ASPIRENF & VALAUT & .20 & .26 & * \\
\hline ASPIRENF & ACOMSCOL & .03 & .33 & ** \\
\hline VALCONF & ATTSOS & .22 & .47 & ** \\
\hline SEF & $\mathrm{NI}$ & -.12 & .21 & ** \\
\hline LECTURE & $\mathrm{NI}$ & .09 & .28 & * \\
\hline TOTCLAS & TOTSCOL & .42 & .33 & * \\
\hline
\end{tabular}

Note de lecture : (dernière ligne) : en milieu populaire, les performances scolaires augmentent de .42 écart-type quand la valeur de l'environnement pédagogique augmente d'un écart-type ; en milieu non populaire, elles augmentent de .33 écart-type dans les mêmes conditions. 
ainsi que le rapport de l'enfant à la lecture sur son niveau intellectuel (0,28 contre 0,09 ns). Enfin, l'effet de l'environnement pédagogique sur les performances scolaires est plus important pour les élèves de milieu populaire $(0,42)$ que pour ceux de milieu non populaire $(0,33)$.

\section{DISCUSSION ET CONCLUSION}

L'hypothèse générale que nous avons cherché à tester, à savoir l'existence de différences intermilieux dans le réseau de causalité des variables en relation avec les performances scolaires, paraît solidement corroborée par cette recherche, compte tenu d'une part de la qualité de l'échantillonnage des familles et de la mesure des principales variables (niveau économico-culturel de la famille, environnement pédagogique, niveau intellectuel et performances scolaires de l'enfant notamment), ainsi que, d'autre part, des méthodes statistiques mises en œuvre pour comparer les deux milieux et des divers contrôles opérés sur les résultats. Cette conclusion doit cependant être nuancée d'une double manière. D'un côté en effet, elle n'est généralisable qu'à la population considérée, caractérisée en particulier par le niveau de scolarité des enfants, et que pour les variables prises en compte et opérationnalisées dans l'enquête (12). D'un autre côté, les différences intermilieux ne concernent pas toutes les relations entre variables et consistent, le plus souvent, en des variations quantitatives ; dit autrement, le réseau de causalité présente de nombreuses similitudes dans les deux milieux comparés, en dépit des différences statistiquement significatives que nous avons pu mettre en évidence.

Cette recherche a pris en compte les différences économiques et culturelles entre les familles d'un même milieu. De nombreux auteurs ont attiré l'attention sur la variabilité intra-milieu (par ex., Périer, 2004, pour le milieu populaire) et nous souscrivons pleinement à leur analyse ; il vaudrait d'ailleurs employer le pluriel et parler, par exemple, de milieux populaires. Cette variabilité interne nous a conduit à introduire l'indice économico-culturel dans le réseau de causalité, alors même que nous souhaitions comparer deux échantillons de familles contrastés selon ce même indice. Or, il s'avère que les différences inter-milieux les plus nombreuses concernent les effets de l'indice. Sans les reprendre toutes, rappelons que les différences de revenu et d'instruction intra-milieu ont un effet plus important sur l'accompagnement de la scolarité dans le milieu populaire, et un effet plus important sur la valorisation de la lecture dans le milieu non populaire. Le fort impact de l'indice économicoculturel peut-il s'expliquer par sa variabilité qui, comme nous l'avons indiqué dans la partie méthodologique, est plus importante dans les milieux non populaires que dans les milieux populaires ? Deux faits s'opposent à cette explication : 1) si l'hétérogénéité infra-milieu était en cause, on devrait constater une régularité dans les différences inter-milieux ; or, ce n'est pas ce que l'on observe : les coefficients de piste sont plus importants, tantôt dans les milieux populaires, tantôt dans les milieux non populaires; 2) lorsque I'on supprime l'indice économico-culturel dans le modèle, les différences inter-milieux subsistent. Les différences observées semblent donc bien indiquer qu'une élévation des ressources financières ou/et du niveau d'instruction des parents n'ont pas les mêmes conséquences sur les attitudes et comportements éducatifs de ceux-ci, selon leur position sur l'échelle des revenus ou/et des niveaux d'étude.

Quel que soit le milieu socio-culturel, l'environnement pédagogique a un effet très important sur les performances scolaires. Rappelons que dans la présente recherche la qualité de l'environnement pédagogique est la résultante de nombreuses variables et qu'elle ne confond pas avec l'efficacité pédagogique du maître. II est par conséquent possible que le rôle de l'environnement pédagogique soit surestimé ici par rapport à d'autres travaux. Mais ce qui est à la fois plus original et plus intéressant, c'est le constat de la non uniformité de cet effet selon l'origine des élèves, ceux des milieux populaires semblant plus sensibles aux variations de l'environnement pédagogique. Si ce résultat était confirmé par des recherches ultérieures, il signifierait que, contrairement à une idée répandue, l'école dispose d'une marge de manœuvre un peu plus élevée pour les enfants des milieux populaires que pour ceux des milieux plus favorisés. Pour éviter tout malentendu, précisons que cette dernière phrase ne signifie pas que l'école soit responsable des différences de réussite liées au milieu social, ni qu'elle puisse facilement les réduire. Elle concerne uniquement la réactivité des élèves de milieu populaire aux caractéristiques des classes et des écoles qu'ils fréquentent. Une amélioration des conditions de prise en charge pédagogique pourrait avoir des conséquences un peu plus bénéfiques pour ces élèves que pour les autres.

En ce qui concerne l'environnement familial, rappelons que nous n'avons pas observé d'effet de l'ac- 
compagnement scolaire parental sur les performances de l'enfant. Ce résultats ne s'accorde pas avec ceux obtenus dans d'autres travaux (cf. Deslandes, 2004, ainsi que Hoover-Dempsey et al., 2001). Mais la recherche de Cooper et al. (1998) suggère que l'accompagnement scolaire n'a pas les mêmes effets à tous les âges. Il est donc possible que l'absence d'effet constatée ici soit due au niveau de scolarité des enfants observés (cours élémentaire). Dans cette étude, ce sont les aspirations scolaires des parents pour leur enfant qui semblent constituer la dimension la plus importante du point de vue des performances scolaires. Par ailleurs, elles ont un effet plus important sur l'accompagnement scolaire dans les familles non populaires que dans les familles populaires. Les attentes des parents envers l'école apparaissent liées à leurs valeurs éducatives générales : les parents qui valorisent la conformité de l'enfant aux normes sociales attendent plus que les autres que l'école remplisse son rôle de socialisation, tandis que ceux qui valorisent l'autonomie de l'enfant attendent davantage qu'elle remplisse sa mission de formation intellectuelle. Mais cette règle générale est modulée par le milieu : les effets de la valorisation du conformisme sur les attentes de socialisation sont nettement plus importants dans les familles des milieux non populaires (il en va de même pour les effets de la valorisation de l'autonomie sur les attentes cognitives, mais la différence inter-milieux n'est pas significative). Tout se passe en quelque sorte comme si les attentes envers l'école étaient davantage influencées par les valeurs éducatives générales en milieu non populaire qu'en milieu populaire. Une dernière remarque porte sur le style éducatif familial dont l'influence sur le niveau intellectuel n'est significative qu'en milieu non populaire. Le faible effet du style éducatif sur le niveau intellectuel est en contradiction avec les résultats obtenus antérieurement par Aubret-Bény (1978) et Manciaux (1975). Cette différence peut peut-être s'expliquer par la faible consistance interne de l'échelle $(0,42)$, ainsi que par la différence des méthodes d'analyse utilisées dans la présente recherche et dans les précédentes, qui ne faisaient pas appel aux pistes causales. Quant à la différence inter-milieux, elle semble refléter une dépendance plus forte du niveau intellectuel à l'égard de l'éducation familiale en milieu non populaire qu'en milieu populaire. L'effet du rapport à la lecture sur le niveau intellectuel, qui ne s'observe qu'en milieu non populaire, va dans le même sens.

II est tentant de mettre en relation les conclusions des deux derniers paragraphes, qui sont d'une certaine manière symétriques. Dans l'ensemble, il semblerait que l'influence du milieu socio-culturel sur les performances scolaires transite davantage par l'école pour les enfants des milieux populaires et davantage par l'éducation familiale et le niveau intellectuel (les deux étant liés) pour les enfants des milieux non populaires. Cette conclusion est évidemment hautement spéculative et demande à être confirmée.

D'une manière générale, cette recherche a produit un ensemble de résultats qui sont à notre connaissance assez nouveaux. Elle montre, également, l'intérêt d'utiliser les MES qui ont la capacité de modéliser un réseau de relations complexes et de comparer ces réseaux entre plusieurs sous-échantillons. Mais son intérêt, à nos yeux, est surtout de suggérer des pistes de recherche nouvelles, qui seront peut-être empruntées par d'autres chercheurs.

Youssef Tazouti

IUFM de Lorraine - GRAPCO, LabPsyLor (Laboratoire de psychologie des universités Nancy 2 et Metz-Paul Verlaine) - Tazouti@univ-nancy2.fr

André Flieller

Université Nancy 2 - GRAPCO, LabPsyLor

(Laboratoire de psychologie des universités Nancy 2 et Metz-Paul Verlaine) - Flieller@univ-nancy2.fr

Pierre Vrignaud

Université Paris X-Nanterre, Laboratoire " Processus cognitifs et conduites interactives" équipe «Développement social et émotionnel » vrignaud.pierre@wanadoo.fr 
(1) HOME est l'acronyme de Home Observation For Measurement of Environment. À l'origine (Bradley \& Cadwell, 1976), l'échelle ne concernait que l'environnement familial des jeunes enfants et reposait à la fois sur l'observation des interactions mère-enfant et sur un questionnaire relatif aux pratiques éducatives parentales. A présent, on dispose de plusieurs versions relatives aux tranches d'âge de zéro à trois ans, de trois à six ans et de six à douze ans. L'échelle a été adaptée en français et révisée par une équipe québécoise (Palacio-Quintin, Jourdan-lonescu \& Lavoie, 1989).

(2) Acronyme pour Linear Structural Relations. Ce modèle fait l'objet d'une presentation dans l'annexe méthodologique.

(3) On peut également se référer à une recherche toute récente du même auteur (Marjoribanks, 2005) faisant apparaître une interaction complexe entre trois variables - le niveau d'aspiration scolaire de l'adolescent, son milieu socio-culturel et son appartenance ethnique - dont les effets sur le niveau d'étude atteint ultérieurement ne sont pas constants.

(4) L'échantillon de 1992 porte, comme celui de 1973, sur une classe d'âge. La majeure partie des élèves fréquentaient le CE1 au début de l'enquête, mais certains étaient scolarisés dans une classe de CP, d'adaptation ou de perfectionnement et un petit nombre d'autres se trouvaient déjà en CE2

(5) Variances respectives de l'indice économico-culturel dans les milieux populaire et non populaire : $s \_1=0,20$; $s \_2=0,66$; différence significative à 0,001 selon le test de Levene; cependant, le coefficient de variation $(\mathrm{s} / \mathrm{m})$ diffère peu d'un milieu à l'autre $(0,15$ et 0,21 respectivement).

(6) Acronyme de Wechsler Intelligence Scale for Children. Ce test bien connu se compose de dix épreuves et fournit trois scores standardisés respectivement pour le total, les épreuves verbales et les épreuves non verbales.
(7) Le lecteur pressé ou peu intéressé par ces informations techniques peut omettre les passages méthodologiques (en plus petits caractères) sans ce que cela nuise à la compréhension des résultats.

(8) Les différences peuvent être attribuées à l'estimation des données manquantes, qui n'est pas effectuée par le même algorithme dans les deux logiciels.

(9) Estimation selon le modèle non contraint (voir l'annexe pour une explication).

(10) Indices d'adéquation au modèle non contraint : $\chi^{2}$ à 40 degrés de liberté $=51.41 ; p=0.11 ;$ RMSEA $=0.02$. Les estimations par LISREL ont été faites dans chaque sous-échantillon, sans modèle d'ensemble, ce qui conduit à des indices d'adéquation différents pour les deux milieux, soit : (1) pour le milieu populaire : $\chi^{2}$ à $20 \mathrm{ddl}=18, p=0,63 ; \operatorname{RMSEA}=0,00 ; \mathrm{GFI}=0,99$; $\mathrm{AGFI}=0,95$; et (2) pour le milieu non populaire : $\chi^{2}$ à $20 \mathrm{ddl}=$ 29, $\mathrm{p}=0,09 ; \mathrm{RMSEA}=0,04 ; \mathrm{GFI}=0,98 ; \mathrm{AGFI}=0,92$. On remarque que l'adéquation est particulièrement bonne pour le sous-échantillon populaire.

(11) Ce sont les coefficients standardisés du modèle non contraint qui sont indiqués dans ce tableau.

(12) Trois limites du modèle théorique peuvent être relevées : 1) il laisse inexpliqués $40 \%$ de la variance des performances ; 2) la mesure de certaines dimensions de l'éducation parentale est sans doute trop sommaire ; 3) le modèle n'adopte pas la perspective interactionniste préconisée par de nombreux auteurs (cf. Bergonnier-Dupuy, 2000).

\section{BIBLIOGRAPHIE}

ARBUCKLE J. L. (2003). AMOS 5.0 Update to the Amos user's guide. Chicago : SmallWaters Corporation.

ARBuckLE J. L. \& WothKe W. (1999). Amos 4.0 user's guide. Chicago: SmallWaters Corporation.

AuBret-BÉnY F. (1978). "Pratiques éducatives des parents et autres caractéristiques culturelles et démographiques du milieu familial, en relation avec le niveau intellectuel des élèves de cours préparatoire ». In Enquête nationale sur le niveau intellectuel des enfants d'âge scolaire. Étude menée conjointement par l'Institut national des études démographiques (INED) \& I'Institut national d'étude du travail et de l'orientation professionnelle (INETOP), Paris : PUF, t. 3, p. 257-291.

Barocas R.; Seifer R.; Sameroff A. J.; Andrews T. A.; CROFT R. T. \& Ostrow E. (1991). "Social and interpersonal determinants of developmental risk ". Developmental Psychology, $\mathrm{n}^{\circ} 27$, p. 479-488.

BÉNÉDETTO P. (1969). "Échelle collective de niveau intellectuel ». In INED et INETOP (éds.), Enquête nationale sur le niveau intellectuel des enfants d'âge scolaire. Paris : PUF, t. 1, p. 33-50

BergonNier-Dupuy G. (2000). "Processus éducatifs intrafamiliaux ; éducation familiale I ». Les sciences de l'éducation pour l'ère nouvelle, t. $33, n^{\circ} 4$, p. 59-81.

Bernstein B. (1975). Langage et classes sociales. Paris : Éd. de Minuit.
BouRdieU P. \& PASSERON J.-C. (1970). La reproduction. Paris : Éd. de Minuit.

Bradley R. H. \& Caldwell B. M. (1976). " The relation of infants' Home environments to mental test performance at 54 months : a follow-up study ». Child Development, vol. $47,1172-1174$.

Bradley R. H. ; Corwyn R. F. ; McAdoo H. P. \& Coll C. G. (2001). "The home environments of children in the United States. Part I : Variations by age, ethnicity, and poverty status ". Child Development, vol. 72, p. 18441867.

Cooper H. ; Lindsay J. J. ; Nye B \& Greathouse S. (1998). "Relationships among attitudes about homework, amount of homework assigned and completed, and student achievement ». Journal of Educational Psychology, vol. 90, p. $70-83$

DESLANDES R. (2004). "Sentiment de compétence parentale et participation parentale dans les travaux scolaires". In R. Toussaint \& C. Xypas (éds.), La notion de compétence en éducation et en formation. Paris : L'Harmattan, p. 275-297.

Duru-Bellat M. \& HenRiot-Van Zanten A. (1992). Sociologie de l'école. Paris : A. Colin.

ESPÉRET E. (1979). Langage et origine sociale des élèves. Bern : P. Lang. 
Flieller A. ; Manciaux M. \& Kop J.-L. (1994). «Évolution des compétences cognitives des élèves en début de scolarité élémentaire sur une période de 20 ans ". In France : ministère de l'Éducation nationale : direction de l'évaluation et de la prospective, L'investissement éducatif et son efficacité : résumé des recherches sélectionnées par l'appel d'offres lancé par la Direction de l'évaluation et de la prospective. Paris : MEN, p. 205-218 (Les dossiers éducation et formations ; $\left.n^{\circ} 47\right)$.

Flieller A., Manciaux M. \& Kop J.-L. (1995). Enquête « 20 ans après »: comparaison de deux cohortes d'écoliers de sept ans, observées à vingt ans d'intervalle (1973-1992). Rapport final. Nancy: université Nancy 2: ADEPS; université Nancy 1-Henri Poincaré : École de santé publique.

Hoover-Dempsey K. ; Battiato A. C. ; Walker J. M. ; Reed R. P. ; DeJong J. M \& JONES K. P. (2001). "Parental involvement in homework ». Educational Psychologist, $n^{\circ} 36$, p. 195-209.

JÖRESKOG K. \& SöRBOM D. (1998). LISREL 8. User's reference guide. Chicago : Scientific Software International.

LAURENS J.-P. (1992). 1 sur 500 : la réussite scolaire en milieu populaire. Toulouse: Presses universitaires du Mirail.

LAUTREY J. (1980). Classe sociale, milieu familial, intelligence. Paris : PUF.

MANCIAUX M. (1975). Enquête sur la prévalence de la débilité mentale dans une population scolaire : étude des caractéristiques socio-professionnelles, familiales et scolaires d'une population d'enfants nés en 1965 en Meurtheet-Moselle. Rapport d'enquête multigraphié. Paris: INSERM.

MARJORIBANKS K. (2001). "Family and ability correlates of academic achievement ». Psychological Reports, vol. 89, p. 510-512.

MARJORIBANKS K. (2003). "Family and ability correlates of academic achievement: Social status group differences ». Psychological-Reports, vol. 93, p. 419-422.

MARJORIBANKS K. (2005). " Family background, adolescents' educational aspirations, and Australian young adult's educational attainment ». International Educational Journal, $\mathrm{n}^{\circ} 6, \mathrm{p} .104-112$.

MULLER J.-L. (1990). « La performance scolaire au cours préparatoire: Les déterminants individuels et familiaux ». Psychologie scolaire, $\mathrm{n}^{\circ} 71$, p. 33-46.

PALACIO-QUiNTIN E. (1995). "Les différences de développement cognitif entre enfants de milieux socio-écono- miques différents et les facteurs associés à ce phénomène ". In J. Lautrey (éd.), Universel et différentiel en psychologie. Paris : PUF, p. 305-325.

Palacio-Quintin E. ; Jourdan-Ionescu C. \& Lavoie T. (1989). "Échelle HOME préscolaire révisée ». Cahiers du GREDEF.

Peterson R. A. (1994). "Cronbach's Alpha Coefficient : A Meta-Analysis ". Journal of Consumer Research, $n^{\circ} 21$, p. 381-391.

PÉRIER P. (2004). «Adolescences populaires et socialisation scolaire : les épreuves relationnelles et identitaires du rapport pédagogique ". L'Orientation scolaire et professionnelle, vol. $33, \mathrm{n}^{\circ} 2$, p. $227-248$.

Pourtois J.-P., Desmet H., Beirens A., Centrella V., Claus S., Gobert F., Nisolle N. \& VANDENBOCh V. (1992). Réussir l'école en milieu pauvre : étude comparative du succès et de l'échec scolaire chez des enfants issus de familles chômeuses et minimexées. Rapport de recherche subsidiée par le ministère de l'Éducation de la Communauté française de Belgique et par le ministère de la programmation de la politique scientifique, document CERIS.

Prêteur Y. \& Louvet-Schmauss E. (1994). «Éducation familiale et acquisition de l'écrit chez des enfants de 5 à 7 ans : une approche comparative franco-allemande ». In M. Deleau \& A. Weil-Barais (éds.), Le développement de l'enfant : approches comparatives. Paris: PUF, p. 244-254.

RocheX J.-Y. (1995). Le sens de l'expérience scolaire : entre activité et subjectivité. Paris : PUF.

Steinberg L. ; Lamborn S. D. ; Dornbusch S. M. \& DarLing N. (1992). " Impact of parenting practices on adolescent achievement: Authoritative parenting, school involvement, and encouragement to succeed ». Child Development, vol. 63, p. 1266-1281.

TAZOUTI Y. (2003). «Éducation familiale et performances scolaires des enfants de milieu populaire ". Revue européenne de psychologie appliquée, $n^{\circ} 53$, p. 97-106.

WothKE W. \& ARBUCKLE J. L. (1996). Full-information missing data analysis with Amos. In F. Faulbaum \& W. Bandilla [éds.] Softstat'95: Advances in statistical software 5. Stuttgart : Lucius.

ZÉroulou Z. (1988). "La réussite scolaire d'enfants immigrés. " Revue française de sociologie, vol. XXIX, $n^{\circ} 3$, p. 447-470. 


\section{ANNEXE MÉTHODOLOGIQUE}

Les modèles d'équations structurales (MES) permettent de modéliser les relations linéaires entre variables. Bien que ces modèles soient aujourd'hui largement utilisés, nous avons jugé utile de rappeler dans cette annexe méthodologique les concepts de base de ces approches (pour des exposés généraux sur les MES, on peut se reporter en français à l'appendice méthodologique de Françoise Bacher dans Reuchlin \& Bacher, 1989). Il est d'usage de distinguer dans les MES le modèle de mesure qui permet d'effectuer une " analyse factorielle confirmatoire » et le modèle structural expliquant les relations entre variables. C'est au modèle structural que l'on s'intéressera ici. Nous exposerons plus en détail la procédure de comparaison de sous-échantillons à l'aide de MES, qui est beaucoup moins connue.

\section{Les modèles en pistes causales}

Le modèle structural proprement dit peut être présenté à partir de la régression multiple qui exprime la relation entre plusieurs variables indépendantes (VI) et une variable dépendante (VD). Les modèles en pistes causales généralisent cette approche à un nombre quelconque de VD. Dans les MES, on peut travailler soit uniquement sur des variables observées (on se situe alors dans le cadre des pistes causales), soit sur des variables latentes et des variables observées (pour une présentation plus détaillée de l'utilisation des MES dans les approches par pistes causales et un exemple, voir Van Acker, Vrignaud \& Lieury, 1997). Par ailleurs, les effets des variables peuvent être « directs » $(A \rightarrow B)$ ou « indirects » $(A \rightarrow B \rightarrow C$, l'effet de $A$ sur $C$ est indirect ou médiatisé, il passe par B). Dans le cas des pistes causales utilisées dans cet article auquel correspond cet article, le modèle comporte quatre types de paramètres: 1) les variables observées ; 2) les coefficients de régression, ou coefficients de piste causale, qui estiment l'effet d'une VI sur une VD ; 3) les covariances, dans le cas où on postule l'existence d'une relation entre une paire de variables mais sans vouloir ou pouvoir faire I'hypothèse d'une relation de causalité entre ces deux variables ; 4) les variances d'erreur, qui sont la part de variance de la VD non expliquée par le modèle (c'està-dire les $\mathrm{VI}$ avec lesquelles elle est en relation).

Les algorithmes d'estimation des MES opèrent sur les matrices de covariances, en conservant la métrique d'origine des variables. II est plus commode pour le chercheur de penser sur des indicateurs stan- dardisés. Par conséquent, les logiciels fournissent des valeurs standardisées pour les coefficients de régression (coefficients de pistes causales), qui sont l'équivalent des coefficients bêta en régression multiple, ainsi que les corrélations lorsqu'on a demandé l'estimation de covariances.

Les modèles en pistes causales permettent d'introduire des effets directs et indirects dans le modèle. Les effets directs correspondent à la valeur du coefficient de régression de la VI sur la VD. On calcule les effets indirects en faisant le produit des coefficients des pistes que l'on doit parcourir pour faire le chemin qui part de la VI et va jusqu'à la VD en passant par des variables intermédiaires, d'où l'appellation de «pistes causales ». Il peut y avoir plusieurs chemins possibles, dans ce cas on fera la somme des effets indirects obtenus en parcourant chacun des chemins possibles. L'effet total d'une variable sur une autre est la somme de l'effet direct (quand il a été introduit dans le modèle) et du/des effet(s) indirect(s), quand il(s) a/ont été introduit(s) dans le modèle. Les logiciels fournissent les valeurs des effets bruts et des effets standardisés.

\section{Test d'un modèle}

Les MES permettent de tester un modèle théorique et d'en estimer les paramètres. Dans le cas des modèles en pistes causales (réseau de relations entre variables observées), la modélisation porte sur la capacité de rendre compte de l'ensemble des relations entre variables par quelques relations causales, justifiées par le chercheur. Le critère d'économie est ici une qualité essentielle du modèle. La qualité d'un modèle sera donc fonction de sa capacité à rendre compte des données et de son caractère économique.

Une fois les paramètres estimés, les logiciels calculent une matrice de covariances estimées. L'écart existant entre cette matrice et celle des covariances observées sert de base au calcul d'un indicateur d'adéquation qui suit asymptotiquement une distribution de $\chi^{2}$; le nombre de degrés de liberté du $\chi^{2}$ est la différence entre le nombre de paramètres dont on demande l'estimation et le nombre de paramètres possibles. Cet indicateur permet de tester l'hypothèse nulle : “ il n'existe pas, dans la population parente, d'écart entre la matrice des covariances estimées et observées. ". On cherche donc à ne pas 
rejeter l'hypothèse nulle puisque c'est l'existence d'une absence d'écart qui permet de considérer que le modèle rend compte des données.

Cette statistique de $\chi^{2}$ présente l'inconvénient d'être sensible à la taille de l'échantillon qui entre dans son calcul. Par ailleurs, les algorithmes utilisés dans les MES (par ex. le maximum de vraisemblance) requièrent des échantillons de taille relativement importante (au moins plusieurs centaines) pour obtenir une estimation robuste des paramètres. Cette dépendance à la taille de l'échantillon augmente fortement le risque de seconde espèce. On a donc voulu pallier cet inconvénient en construisant des indicateurs moins sensibles à la taille de l'échantillon, plus faciles à interpréter (ayant un intervalle de variation connu) et également peu sensibles au nombre de paramètres. Cette recherche a abouti à un grand nombre d'indicateurs. La difficulté, voire l'impossibilité dans certains cas, d'établir la distribution de ces indicateurs ainsi que leur robustesse à différentes conditions a donné lieu à de nombreuses études de simulation (par ex. Hu \& Bentler, 1999). On peut classer les indicateurs en différentes familles selon le rationnel qui a présidé à leur construction. Pour une présentation détaillée, on se reportera soit à un ouvrage général comme celui de Bollen (1989), soit au manuel des logiciels (par ex., Arbuckle \& Wothke, 2003 ; Jöreskog, \& Sörbom, 1998).

\section{Les indicateurs basés sur l'écart au modèle d'indépendance}

Ces indicateurs se basent sur le rapport entre le $\chi^{2}$ du modèle testé et le $\chi^{2}$ du modèle d'indépendance, modèle où l'on fait l'hypothèse qu'il n'existe pas de relations entre les variables (il s'agit en quelque sorte du pire des modèles). La valeur des indicateurs basés sur l'écart au modèle d'indépendance peut varier théoriquement de 0 à 1 . On considère qu'ils indiquent un bon ajustement à partir de valeurs de l'ordre de 0,90 . Cependant les études de simulation tendent à montrer qu'il vaut mieux retenir des valeurs plus élevées de l'ordre de 0,95, voire 0,97. Dans cette famille, nous avons retenu le TLI (Tucker \& Lewis Index).

\section{Les indicateurs basés sur l'écart observé/estimé}

Le RMSEA (Root Mean Square Error of Approximation) est l'un des indicateurs les plus recommandés (Browne \& Cudeck, 1993) car il est indépendant de la taille de l'échantillon et du nombre de degrés de liberté du modèle. De ce fait, son intervalle de varia- tion est comparable d'un échantillon et d'un modèle à l'autre. La plupart des auteurs considèrent qu'un modèle présentant une valeur supérieure à .08 devrait être amélioré et qu'une valeur supérieure à .10 est inacceptable. Une valeur inférieure à .05 traduit un bon ajustement. Ces seuils ont été, en grande partie, confirmés par les études de simulation.

\section{Les indicateurs basés sur la variance expliquée}

Le GFI (Goodness of Fit Index) rend compte de la proportion de variance de la matrice observée expliquée par le modèle. L'AGFI (Adjusted Goodness of Fit Index) corrige le GFI pour le nombre de degré de liberté. Ces indicateurs varient théoriquement entre 0 et 1 . Un bon ajustement se traduit par des valeurs au moins égales à .90. Mais, comme pour les indicateurs basés sur l'écart au modèle d'indépendance, il vaut mieux considérer des valeurs plus élevées.

Une règle de bonne hygiène [théorique/méthodologique/épistémologique] pour l'interprétation des indicateurs des MES consiste, comme le conseillent d'ailleurs les auteurs d'études de simulation, à s'appuyer sur plusieurs indicateurs simultanément $(\mathrm{Hu} \&$ Bentler, 1999).

Ces indicateurs répondent à la question de l'adéquation globale du modèle. Ils permettent de vérifier que les relations introduites dans le modèle rendent compte, prises dans leur ensemble, des relations observées dans les données. II est souhaitable de s'intéresser également à chacun des coefficients de piste. On utilise alors un test local obtenu en faisant le rapport entre la valeur du paramètre (par ex. le coefficient de piste causale) et son erreur type d'estimation (valeur fournie par l'algorithme d'estimation). Cette statistique suit une distribution de $Z$ et permet de tester l'hypothèse nulle : " la valeur du paramètre est égale à zéro dans la population parente. ". On cherche ici à rejeter l'hypothèse nulle pour confirmer l'existence du paramètre dans le modèle.

\section{Comparaison de modèles et de groupes}

La démarche de test de l'adéquation d'un modèle théorique peut être étendue à la comparaison de l'adéquation de deux ou plusieurs modèles. On va comparer la plus ou moins bonne adéquation de chacun des modèles aux données. Dans le cas où les modèles sont emboîtés - deux modèles sont emboîtés si on peut passer de l'un à l'autre en retirant ou en ajoutant des pistes causales - on obtiendra un test de 
I'hypothèse selon laquelle un modèle donné présente une meilleure adéquation aux données qu'un modèle concurrent. On teste cette hypothèse en faisant la différence entre les valeurs des $\chi^{2}$ des modèles comparés, le nombre de degrés de liberté étant égal à la différence entre le nombre de degrés de liberté du modèle emboîtant et celui du modèle emboîté. Cette statistique permet de tester l'hypothèse nulle : «Dans la population parente, il n'existe pas de différence entre les modèles pour rendre compte des relations entre variables. ". On cherche, bien sûr, à rejeter cette hypothèse nulle, ce qui permet de conclure que le modèle emboîtant apporte une amélioration significative par rapport au modèle emboîté.

La comparaison de groupes va être conduite comme une comparaison de modèles. L'estimation des paramètres sera effectuée, non plus sur une seule matrice de covariances, mais en utilisant, conjointement, une matrice par sous-échantillon. Rappelons qu'il est possible d'introduire des contraintes sur l'estimation des paramètres, le plus souvent, des contraintes d'égalité. Comparer des groupes revient à comparer un modèle dit non contraint, dans lequel l'algorithme estime des valeurs spécifiques des paramètres pour chacun des groupes, et un modèle contraint où les valeurs de chacun des paramètres sont contraintes à l'égalité entre les groupes. S'il existe des différences entre les groupes, alors on s'attend à ce que le modèle non contraint présente une amélioration significative de l'adéquation aux données par rapport au modèle contraint. Comme le modèle contraint est emboîté dans le modèle non contraint, on testera cette hypothèse en faisant la différence entre les $\chi^{2}$ de ces deux modèles. Si le test de la différence est significatif, on peut considérer que les paramètres des pistes causales n'ont pas la même valeur dans les sous-échantillons. Pour affiner cette hypothèse globale, on peut tester les différences entre chaque paire de paramètres, ici entre les valeurs du même paramètre dans chacun des groupes. Ce test est calculé en rapportant la différence entre les valeurs des paramètres à l'erreur type de mesure de cette différence. II suit une distribution de $Z$.

\section{BIBLIOGRAPHIE DE L'ANNEXE}

ARBUCKLE J. L. (2003). AMOS 5.0 Update to the Amos user's guide. Chicago : SmallWaters Corporation.

BOLLen K. A. (1989). Structural equation with latent variables. New York : Wiley.

Browne M. W. \& CUDECK R. (1993). «Alternative ways of assessing model fit ». In K. A. Bollen \& J. S. Long (éds.), Testing structural equation models. London : Sage, p. 136-162.

Hu L. \& BentLeR P. M. (1999). « Cutoff criteria for fit indexes in covariance structure analysis : Conventional criteria versus new alternatives". Structural Equation Modeling, $\mathrm{n}^{\circ} 6$, p. 1-55.
JöRESKOG K. \& SöRBOM D. (1998). LISEL 8. User's reference guide. Chicago : Scientific Software International.

REUCHLIN M. \& BACHER F. (1989). Les différences individuelles dans le développement cognitif de l'enfant. Paris : PUF.

Van Acker P. ; Vrignaud P. \& Lieury A. (1997). " Mémoire de travail, mémoire encyclopédique et performance scolaire en $3^{\mathrm{e}}$ ». L'Orientation scolaire et professionnelle, $\mathrm{n}^{\circ} 26$, p. $571-596$.

WothKe W. \& ARBUCKLE J. L. (1996). « Full-information missing data analysis with Amos". In F. Faulbaum et W. Bandilla (éds.). Softstat '95: Advances in statistical software 5. Stuttgart : Lucius. 